\title{
First-principles investigation of pentagonal and hexagonal core-shell silicon nanowires with various core compositions
}

\author{
C. Berkdemir ${ }^{1}$ and O. Gülseren ${ }^{2, *}$ \\ ${ }^{1}$ Department of Physics, Erciyes University, Kayseri 38039, Turkey \\ ${ }^{2}$ Department of Physics, Bilkent University, Ankara 06800, Turkey
}

(Received 13 August 2008; published 30 September 2009)

\begin{abstract}
Properties of various core-shell silicon nanowires are investigated by extensive first-principles calculations on the geometric optimization as well as electronic band structures of the nanowires by using pseudopotential plane-wave method based on the density-functional theory. We show that different geometrical structures of silicon nanowires with various core compositions, formed by stacking of atomic polygons with pentagonal or hexagonal cross sections perpendicular to the wire axis, can be stabilized by doping with various types of semiconductor ( $\mathrm{Si}, \mathrm{Ge}$ ), nonmetal (C), simple metal (Al), and transition metal (TM), $3 d$ (Ti, Cr, Fe, Co, Ni, $\mathrm{Cu}), 4 d(\mathrm{Nb}, \mathrm{Mo}, \mathrm{Pd}, \mathrm{Ag})$, and $5 d(\mathrm{Ta}, \mathrm{W}, \mathrm{Pt}, \mathrm{Au})$, core atoms. Dopant atoms are fastened to a linear chain perpendicular to the planes of Si-shell atoms and are located through the center of planes. According to the stability and energetics analysis of core-shell Si nanowires, the eclipsed pentagonal and hexagonal structures are energetically more stable than the staggered ones. Electronic band structure calculations show that the pentagonal and hexagonal Si-shell nanowires doped with various different types of core atoms exhibit metallic behavior. Magnetic ground state is checked by means of spin-polarized calculations for all of the wire structures. The eclipsed hexagonal structure of Si-shell nanowire doped with Fe atom at the core has highest local magnetic moment among the magnetic wire structures. Electronic properties based on band structures of Si-shell nanowires with different dopant elements are discussed to provide guidance to experimental efforts for silicon-based spintronic devices and other nanoelectronic applications.
\end{abstract}

DOI: 10.1103/PhysRevB.80.115334

PACS number(s): 73.22.-f, 61.46.Km, 62.23.Hj, 61.72.U-

\section{INTRODUCTION}

Over the last decade, silicon nanowires (SiNWs) have obtained broad attention for possible use in integrated nanoscale electronics ${ }^{1-3}$ as well as for studying fundamental properties of structures and devices with very small dimensions. ${ }^{4}$ Their electrical and optical properties ${ }^{5}$ have been widely investigated to control their growth directions to synthesize these nanowires. ${ }^{6-11}$ Moreover, semiconductor nanowires are very important as critical building blocks for electronic devices such as field-effect and thin-film transistors. ${ }^{11-14}$ For example, it has been suggested that the SiNWs thinner than $100 \mathrm{~nm}$ in diameter might be used in light-emitting devices with extremely low power consumption ${ }^{15}$ and in Schottky barrier field-effect transistors. ${ }^{16}$ However, for integration of these nanoelectronic devices, it is necessary to connect different nanotubes and nanowires to form the source-drain doping and metal contacts. Moreover, contact metals might account as an alternative to traditional doped source-drain device structures where one suffers from fundamental problems such as high leakage current and parasitic resistance arise from the sub-100 $\mathrm{nm}$ range scaling. On the contrary, silicon nanowire, ${ }^{17}$ or nanotube ${ }^{18-22}$ itself might be incorporated as nanocontact structure. Although the stability of various silicon nanotubes (SiNTs) has been verified from computational studies, ${ }^{18-22}$ and energetics of SiNTs, for example dependence of strain energy on the tube diameter and chirality, is studied in detail, ${ }^{23}$ it is very difficult to realize them experimentally because of $s p^{3}$ hybridization tendency of silicon in SiNTs. On the other hand, SiNWs, which are more stable than SiNTs, might benefit from this hybridization for its stabilization. As a summary, SiNWs are of both funda- mental and technological interest, and they have been made a range of one-dimensional nanostructures. However, largescale fabrication of these nanowires is still a challenge, and consequently, experimental ${ }^{24}$ and first-principle ${ }^{25}$ architectures are still required.

In spite of the limited experimental studies on the structures, theoretical investigations on the electronic structures, mechanical properties, and uniaxial-stress effects of different types of nanowires are currently performed using firstprinciples calculations. Numerous theoretical and computational studies on SiNW have been published in recent years. ${ }^{26-30}$ For example, the most stable geometries for pristine SiNWs grown along their (100) axis, ${ }^{31}$ and the effects of different surface species on the band gap of $\mathrm{SiNWs}^{32}$ are determined by an exhaustive stability analysis. The quantum conductivity, structural stability, and optical properties of small diameter SiNWs are investigated ${ }^{33,34}$ by considering tetrahedral, cagelike and polycrystalline wires. Furthermore, first-principles calculations have been performed to investigate Si-based nanostructures, mainly including tricapped and uncapped trigonal prisms, pristine silicon whiskers and single-walled silicon nanotubes with different diameters and chiral vectors. ${ }^{35,36}$ The effect of wire thickness on the band gap, conduction valley splitting and hole band splitting have been demonstrated using a single-band effective mass model. ${ }^{37}$ Similarly, effect of terminating the nanowire surfaces by hydrogen atoms is also discussed. ${ }^{38}$ Despite these recent efforts and studies, there is still a matter of debate about the production of SiNWs and the most stable structures of small diameter SiNWs. For instance, nanowires derived from the silicon clathrate phases are predicted to be more stable $\mathrm{e}^{39,40}$ and energetically more favorable than the diamond 
type of SiNWs at the same diameters. ${ }^{41}$ However, pristine clathrates are semiconductors and have wider band gaps than that of the diamond phase of silicon. ${ }^{42}$ Nevertheless, electronic character of clathrate types of nanowires which are consisted of 30 to $36 \mathrm{Si}$ atoms in its primitive unit cell ranges from semiconducting to metallic. ${ }^{43}$ These types of nanowires are intercalated with alkali and alkaline-earth metals as well.

With the experimental developments, pentagon-shaped silicon nanowires with linewidth around $300 \mathrm{~nm}$ are successfully fabricated by using the $\mathrm{Si} / \mathrm{SiGe}$ epitaxy technique and etching mechanisms. ${ }^{6}$ As a key point in fabrication of SiNWs, study of SiNWs of various sizes and shapes is now a focus of interest of theoretical studies seeking more fundamental understanding of all these nanowire structures. ${ }^{44,45}$ Along these lines, the synthesis ${ }^{46}$ of metal encapsulating $\mathrm{Si}$ clusters provides important clues for realization of their onedimensional infinite analogs. The stability and several properties of these Si cage structures consisting of various types of metal atoms have been studied in detail by $a b$ initio calculations. ${ }^{47-51}$ Henceforth, the stability of finite and infinite hexagonal prismatic structures of $\mathrm{Si}$ has been checked through doping with various transition metal (TM) atoms $^{52-56}$ in order to identify the concrete structures of SiNWs. For example, Menon et al. ${ }^{52}$ have examined the various cagelike structures of $\mathrm{Si}$ stabilized by encapsulation of $\mathrm{Ni}$, and the stability of the infinite Ni-Si nanotube structure from tight-binding molecular dynamics and $a b$ initio calculations. The infinite nanotube derived from cagelike structures has been generated from a unit cell consisting of $4 \mathrm{Ni}$ and $20 \mathrm{Si}$ atoms. Similarly, Andriotis et al. ${ }^{53}$ have shown that the encapsulation of metal atoms, Ni and V, within Si-based cage clusters leads to stable metal-encapsulated Si cage clusters and nanotubes. It has been also found that the magnetic moment of the Si-encapsulated $\mathrm{Ni}$ or $\mathrm{V}$ atom is much less than the corresponding value of the free atom. Dumitrica et $a l{ }^{54}$ have described how the smallest silicon nanotubes with $(2,2)$ and $(3,0)$ chiral symmetries are stabilized by the axially placed metal atoms. Singh et al. ${ }^{55}$ have shown that hexagonal metallic silicon nanotubes can be stabilized by doping with TM-3d atoms, besides Fe-doped nanotube has large magnetic moment per Fe atom, nearly the same as in bulk Fe. Moreover, Jang et al. ${ }^{56}$ have investigated magnetic properties of $\mathrm{Fe}-, \mathrm{Co}-$ and $\mathrm{Ni}$-doped infinite silicon nanotubes with hexagonal prism structure for two different numbers of dopants. Likewise, Durgun et al. ${ }^{21}$ have explored whether various structures doped with TM atoms in the pursuit of finding the energetically most favorable units can be generated by stacking of triangle, pentagons, or hexagons of Si.

In order to realize and fabricate the Si nanowires, first of all, it is essential to understand the nanowire structure, i.e., various shapes and sizes as well as the effect of different dopants. Moreover, doping with various elements, especially with TM atoms because of $d$ band filling, besides stabilizing the structure, could lead to an entirely new range of siliconbased applications in nanoelectronic and spintronics devices based on metallic properties of SiNWs. Even though, the studies we reviewed above indicate that various TM atoms might stabilize the cagelike Si clusters and the finite-infinite tubular structures of $\mathrm{Si}$, it is necessary to have a systematic investigation of the effect of various core structures with a) EP-top view

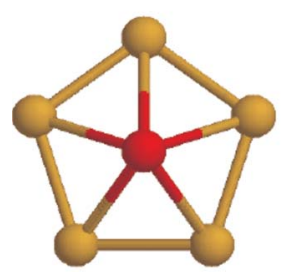

c) SP-top view

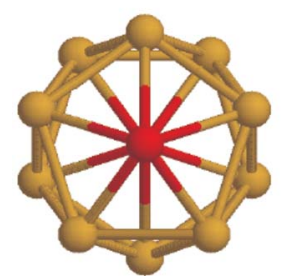

b) EP-side view

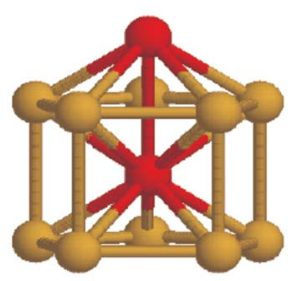

d) SP-side view

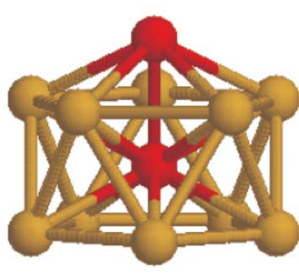

FIG. 1. (Color online) (a) Top view of EP core-shell SiNW; light (yellow) and dark (red) balls denote $\mathrm{Si}$ atoms and $M$ atoms at the core, respectively. (b) Side view of EP core-shell SiNW. (c) Top view of SP core-shell SiNW; successive pentagons are rotated by $\pi / 5$. (d) Side view of SP core-shell SiNW.

different atoms on nanowire structure stability, and there are still some open questions on the magnetic properties and electronic band structures of core-shell SiNWs with various core compositions, such as semiconductor, nonmetal, simple metal as well as TM atoms. The central point of this paper is to address these questions from extensive first-principles calculations within the density-functional theory (DFT). We consider core-shell SiNWs with two different geometries which are shaped as pentagonal (see Fig. 1) and hexagonal (see Fig. 2) in cross-section perpendicular to the wire axis. Each of these geometries are also separated with two subgeometries which are called as eclipsed and staggered.

a) EH-top view

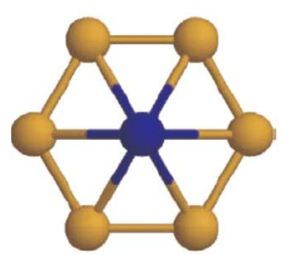

c) SH-top view

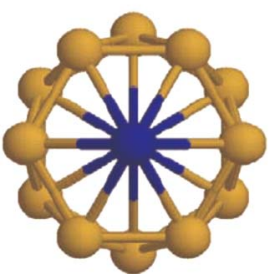

b) EH-side view

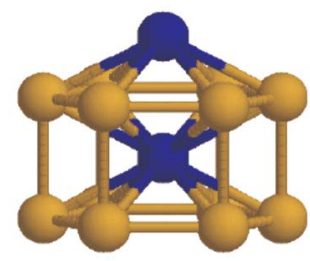

d) SH-side view

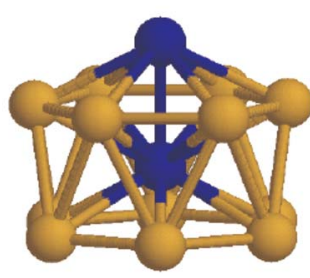

FIG. 2. (Color online) (a) Top view of EH core-shell SiNW; light (yellow) and dark (blue) balls denote $\mathrm{Si}$ and $M$ atoms at the core, respectively. (b) Side view of EH core-shell SiNW. (c) Top view of SH core-shell SiNW; successive hexagons are rotated by $\pi / 6$. (d) Side view of SH core-shell SiNW. 
We carry out state-of-the-art total energy calculations for $M(M=\mathrm{C}, \mathrm{Si}, \mathrm{Ge}, \mathrm{Al}, \mathrm{Ti}, \mathrm{Cr}, \mathrm{Fe}, \mathrm{Co}, \mathrm{Ni}, \mathrm{Cu}, \mathrm{Nb}, \mathrm{Mo}, \mathrm{Pd}$, $\mathrm{Ag}, \mathrm{Ta}, \mathrm{W}, \mathrm{Pt}, \mathrm{Au}$ ) atoms fastened to the monatomic chain passing through the centers of the parallel polygons [eclipsed pentagon (EP) and staggered pentagon (SP) as shown in Fig. 1 , eclipsed hexagon $(\mathrm{EH})$ and staggered hexagon $(\mathrm{SH})$ as presented in Fig. 2]. We explored the most stable structures of $\mathrm{Si}$-shell nanowires with various types of geometries. We compute the electronic band structures to reveal the origin of stability and electronic properties of the polygonal structures. We also discuss the effect of both pentagonal and hexagonal structures on the ballistic conductance by examining the electronic bands crossing the Fermi energy. Moreover, using the first-principles calculations, we investigate the magnetic properties of Si-shell nanowires doped with $M$ atoms (magnetic and nonmagnetic transition metals) for all geometrical structures.

\section{COMPUTATIONAL METHODS}

All calculations in this study are performed within the framework of DFT (Ref. 57) by first-principles plane-wave method $^{58,59}$ by using ultrasoft Vanderbilt pseudopotentials. ${ }^{60}$ Both local density approximation (LDA) (Ref. 61) and generalized gradient approximation (GGA) (Ref. 62) is explored for the exchange-correlation energy. The isolated nanowires are described within the supercell geometry using a tetragonal unit cell. The axis of the core-shell SiNW is taken along the $z$ axis, and the lattice parameter of the SiNW coincides with the lattice parameter $c$ of the tetragonal supercell. To minimize the interaction between a SiNW and its periodic images, the lattice parameters of the tetragonal cell in the $x-y$ plane are taken to be $a=b=18 \AA$. The cutoff energy of 400 $\mathrm{eV}$ (29.4 Ry) for plane-wave expansion is found to be sufficient after the convergence tests. Methfessel-Paxton smearing method ${ }^{63}$ is used to treat the partial occupancies and the width of the smearing is $0.06 \mathrm{eV}$. For the Brillouin zone integrations, $1 \times 1 \times 45 \mathrm{k}$-point mesh according to the Monkhorst-Pack scheme ${ }^{64}$ is used for the one-dimensional infinite SiNWs. On the other hand, only the $\Gamma$ point is used for the case of the finite structure (i.e., for atomic energies). The exchange-correlation potential is approximated by GGA (Ref. 62) for full relaxed atomic structures. The conjugate gradient (CG) method is used for wave-function optimization, where the total energy and atomic forces are minimized. In order to check the correct ground state, we also performed spin-polarized calculations for core-shell SiNWs.

\section{RESULTS AND DISCUSSIONS}

The pentagonal and hexagonal structures considered are illustrated in Figs. 1 and 2, respectively. The atomic polygons shown in Fig. 1 are made of pentagon of silicon atoms, i.e., shell structure, which are perpendicular to the $z$ axis of the nanowire with separation $w$. In EP, parallel pentagons are identical and located in an eclipsed (top-to-top) position; in SP, successive pentagons are rotated by $\pi / 5$ and placed in a staggered position. In order to construct the nanowire, a $M$ atom as a linear chain along the $z$ axis is passed through the center of pentagons and each chain atom is fastened to a point equidistance from the layers of pentagons. Note that the geometrical structures of these core-shell SiNWs have different structural parameters. The lattice parameter $c$ of EP SiNWs equals to separation $w$, i.e., $c=w$, but in the case of SP SiNWs, the $c$ is twice the spacing as the staggered pentagons, i.e., $c=2 w$. Accordingly, EP and SP structures contain 6 and 12 atoms within the unit cell, respectively. Similarly, examples of the top and side views of hexagonal structures are shown in Fig. 2. The definitions described above for labeling the pentagonal SiNWs are also valid for hexagonal structures, but in this case, successive hexagons are rotated by $\pi / 6$. In addition, the number of atoms in the unit cell is 7 and 14 for $\mathrm{EH}$ and $\mathrm{SH}$ structures, respectively.

\section{A. Optimized structures and energetics}

The energetics and atomic structures of pentagon and hexagon-shaped SiNWs doped with $M$ atoms have been reported to confirm the stability of SiNWs. The averaged binding energy $E_{b}$ (magnetic or nonmagnetic) per atom for our nanowire structures is calculated from the following expression:

$$
E_{b}=\left[N_{\mathrm{Si}} E_{a}(\mathrm{Si})+N_{M} E_{a}(M)-E_{T}(\mathrm{SiNW})\right] / N_{t},
$$

where $E_{a}(\mathrm{Si})$ and $E_{a}(M)$ are the energies of single $\mathrm{Si}$ and $M$ atoms, respectively, and $E_{T}(\mathrm{SiNW})$ is the optimized total energy of a core-shell SiNW. $N_{\mathrm{Si}}$ and $N_{M}$ are the numbers of Si and $M$ atoms, respectively, so that $N_{t}=N_{\mathrm{Si}}+N_{M}$ is the total number of atoms in the unit cell. Hence, positive $E_{b}$ means the stability of SiNW with respect to the constituent atoms. Our results for the interatomic bond lengths $\ell$ and binding energies $E_{b}$ as well as the number of bands crossing the Fermi level of optimized structures of core-shell SiNWs are compiled in Table I. The bond length between two adjacent $\mathrm{Si}$ atoms located on the planes of pentagons or hexagons is shown with $\ell_{\mathrm{Si}-\mathrm{Si}}$. The nearest distance between two neighboring $\mathrm{Si}$ atoms on the separated pentagons or hexagons is denoted with $\widetilde{\ell}_{\mathrm{Si}-\mathrm{Si}}$. It is noted that a $\mathrm{Si}$ atom situated at the top planes of EP or EH structures is connected by a vertical line to the other partner at the down ones whereas the same atom at SP or SH structures is connected by a zigzag line. The bond length between two $M$ atoms is represented with $\ell_{M-M}$. These lengths are a scale of the spacing between adjacent pentagons and hexagons as well. By this means, $\ell_{M-M}$ is equal to the lattice constant $c$ for the eclipsed structures, i.e., $\ell_{M-M}=c$, but in the staggered ones, the lattice constant $c$ is twice $\ell_{M-M}$, i.e., $c=2 \ell_{M-M}$. Last, the bond length between $\mathrm{Si}$ and $M$ atoms is denoted by $\ell_{\mathrm{Si}-M}$. The binding energies and the relevant interatomic bond lengths corresponding to equilibrium bulk crystal structures, calculated with the same computational parameters used in nanowire systems, are also presented for the sake of comparison.

Comparison of the calculated averaged binding energies presented in Table I shows that for the case of pentagonalshaped SiNWs the eclipsed pentagonal structures are energetically more favorable than staggered ones for all of the considered elements except $\mathrm{Ti}$ and $\mathrm{Cr}$. However, the staggered pentagonal structure for $\mathrm{Ti}$ and $\mathrm{Cr}$ has the binding 
TABLE I. Optimized structural parameters and the binding energy $E_{b}$ (either magnetic or nonmagnetic) of core-shell SiNWs. Bond lengths and energies are in $\AA$ and $\mathrm{eV}$, respectively. Two different bond length separated by a slash (/) means a nonuniform structure. For bulk diamond $\mathrm{Si}$, the cohesive energy is calculated as $5.43 \mathrm{eV}$ while the $\mathrm{Si}-\mathrm{Si}$ bond length is calculated as $2.37 \AA$. The number of bands crossing the Fermi level (conductance channel numbers for the perfect contact case) of SiNWs are given by $\eta$. For magnetic cases, first reported conductance channel number is for majority spins while the second one is for minority spins.

\begin{tabular}{|c|c|c|c|c|c|c|c|c|c|c|c|c|c|}
\hline \multirow[b]{2}{*}{ Atom } & \multirow[b]{2}{*}{ Str. } & \multicolumn{6}{|c|}{ Pentagonal $(\mathrm{P})$ geometry } & \multicolumn{6}{|c|}{ Hexagonal $(\mathrm{H})$ geometry } \\
\hline & & $\ell_{\mathrm{Si}-\mathrm{Si}}$ & $\tilde{\ell}_{\mathrm{Si}-\mathrm{Si}}$ & $\ell_{\mathrm{Si}-M}$ & $\ell_{M-M}$ & $\begin{array}{c}E_{b} \\
(\mathrm{eV})\end{array}$ & $\eta$ & $\ell_{\mathrm{Si}-\mathrm{Si}}$ & $\tilde{\ell}_{\mathrm{Si}-\mathrm{Si}}$ & $\ell_{\mathrm{Si}-M}$ & $\ell_{M-M}$ & $\begin{array}{c}E_{b} \\
(\mathrm{eV})\end{array}$ & $\eta$ \\
\hline $\mathrm{Si}$ & $\mathrm{S}$ & 2.59 & 2.97 & 2.57 & 2.64 & 4.473 & 6 & 2.53 & 2.71 & 2.80 & 2.37 & 4.523 & 9 \\
\hline $\mathrm{Si}$ & $\mathrm{E}$ & 2.52 & 2.76 & 2.55 & 2.76 & 4.544 & 10 & $2.38 / 2.54$ & 2.54 & $2.92 / 2.50$ & 2.54 & 4.622 & 6 \\
\hline $\mathrm{Ge}$ & $\mathrm{S}$ & 2.67 & 3.07 & 2.65 & 2.74 & 4.283 & 6 & $2.62 / 2.59$ & 2.79 & 2.87 & 2.44 & 4.334 & 9 \\
\hline $\mathrm{Ge}$ & $\mathrm{E}$ & 2.59 & 2.85 & 2.62 & 2.85 & 4.288 & 10 & $2.44 / 2.56$ & 2.60 & $2.93 / 2.65$ & 2.60 & 4.451 & 4 \\
\hline $\mathrm{C}$ & $\mathrm{S}$ & 2.37 & 2.85 & 2.39 & 2.56 & 4.652 & 6 & 2.45 & 2.59 & 2.70 & 2.26 & 4.555 & 5 \\
\hline $\mathrm{C}$ & $\mathrm{E}$ & 2.34 & 2.60 & 2.37 & 2.60 & 4.812 & 6 & 2.37 & 2.48 & 2.67 & 2.48 & 4.681 & 3 \\
\hline $\mathrm{C}$ & $\mathrm{E}$ & & & & & & & $2.44 / 2.47$ & 2.66 & $3.05 / 1.87$ & 2.66 & 4.940 & 3 \\
\hline $\mathrm{Al}$ & S & 2.70 & 2.89 & 2.62 & 2.51 & 4.290 & 6 & 2.49 & 2.79 & 2.79 & 2.48 & 4.394 & 7 \\
\hline $\mathrm{Al}$ & $\mathrm{E}$ & 2.57 & 2.72 & 2.58 & 2.72 & 4.356 & 9 & 2.43 & 2.63 & 2.76 & 2.63 & 4.502 & 5 \\
\hline $\mathrm{Ti}$ & $S$ & 3.00 & 2.75 & 2.60 & 2.06 & 4.965 & 6 & 2.50 & 2.79 & 2.79 & 2.47 & 5.084 & 7 \\
\hline $\mathrm{Ti}$ & $\mathrm{E}$ & 2.74 & 2.52 & 2.65 & 2.51 & 4.916 & 10 & 2.49 & 2.47 & 2.78 & 2.47 & 5.118 & 7 \\
\hline $\mathrm{Cr}$ & $\mathrm{S}$ & 2.87 & 2.53 & 2.64 & 2.15 & 5.383 & 10 & 2.44 & 2.69 & 2.70 & 2.38 & 5.359 & 5 \\
\hline $\mathrm{Cr}$ & $\mathrm{E}$ & 2.59 & 2.46 & 2.52 & 2.46 & 5.369 & 3 & 2.41 & 2.43 & 2.70 & 2.43 & 5.389 & 9 \\
\hline $\mathrm{Cr}$ & $\mathrm{E}$ & 2.59 & 2.46 & 2.52 & 2.46 & 3.843 & $7(\uparrow) / 3(\downarrow)$ & & & & & & \\
\hline $\mathrm{Fe}$ & S & 2.72 & 2.61 & 2.56 & 2.18 & 4.116 & $3(\uparrow) / 4(\downarrow)$ & $2.42 / 2.41$ & 2.71 & $2.71 / 2.68$ & 2.40 & 4.121 & $4(\uparrow) / 2(\downarrow)$ \\
\hline $\mathrm{Fe}$ & $\mathrm{E}$ & 2.47 & 2.56 & 2.46 & 2.56 & 5.292 & 6 & & & & & & \\
\hline $\mathrm{Fe}$ & $\mathrm{E}$ & 2.54 & 2.48 & 2.49 & 2.48 & 4.155 & $6(\uparrow) / 3(\downarrow)$ & 2.40 & 2.47 & 2.70 & 2.47 & 4.196 & $3(\uparrow) / 4(\downarrow)$ \\
\hline Co & $\mathrm{S}$ & 2.76 & 2.59 & 2.58 & 2.15 & 4.284 & $4(\uparrow) / 3(\downarrow)$ & 2.36 & 2.74 & 2.66 & 2.45 & 4.223 & $6(\uparrow) / 4(\downarrow)$ \\
\hline Co & $\mathrm{S}$ & & & & & & & $2.39 / 2.38$ & $2.72 / 2.74$ & $2.70 / 2.61$ & 2.43 & 4.224 & $6(\uparrow) / 4(\downarrow)$ \\
\hline $\mathrm{Co}$ & $\mathrm{E}$ & 2.48 & 2.54 & 2.46 & 2.54 & 4.376 & $7(\uparrow) / 5(\downarrow)$ & $2.36 / 2.40$ & 2.46 & $2.65 / 2.75$ & 2.46 & 4.309 & $8(\uparrow) / 5(\downarrow)$ \\
\hline $\mathrm{Ni}$ & $S$ & 2.73 & 2.61 & 2.57 & 2.18 & 4.838 & 6 & 2.40 & 2.72 & 2.70 & 2.42 & 4.815 & 5 \\
\hline $\mathrm{Ni}$ & $\mathrm{E}$ & 2.51 & 2.51 & 2.47 & 2.51 & 4.940 & 5 & 2.38 & 2.46 & 2.68 & 2.46 & 4.925 & 9 \\
\hline $\mathrm{Cu}$ & $\mathrm{S}$ & 2.78 & 2.68 & 2.61 & 2.24 & 4.398 & 8 & $2.44 / 2.57$ & $2.61 / 2.82$ & $2.73 / 2.78$ & 2.40 & 4.444 & 8 \\
\hline $\mathrm{Cu}$ & $\mathrm{E}$ & 2.54 & 2.57 & 2.51 & 2.54 & 4.503 & 3 & 2.42 & 2.48 & 2.72 & 2.48 & 4.604 & 5 \\
\hline $\mathrm{Nb}$ & $\mathrm{S}$ & 2.80 & 2.89 & 2.71 & 2.60 & 5.214 & 9 & 2.49 & 2.92 & 2.81 & 2.62 & 5.425 & 5 \\
\hline $\mathrm{Nb}$ & $\mathrm{S}$ & & & & & & & $2.52 / 2.47$ & $2.92 / 2.88$ & $2.80 / 2.83$ & 2.61 & 5.425 & 5 \\
\hline $\mathrm{Nb}$ & E & 2.79 & 2.60 & 2.71 & 2.60 & 5.317 & 9 & 2.50 & 2.57 & 2.81 & 2.57 & 5.462 & 10 \\
\hline Mo & S & 2.76 & 2.88 & 2.66 & 2.49 & 5.444 & 5 & 2.49 & 2.82 & 2.79 & 2.52 & 5.546 & 3 \\
\hline Мо & $\mathrm{E}$ & 2.72 & 2.56 & 2.64 & 2.56 & 5.536 & 5 & 2.47 & 2.52 & 2.77 & 2.52 & 5.592 & 10 \\
\hline $\mathrm{Pd}$ & $\mathrm{S}$ & 2.61 & 3.05 & 2.61 & 2.72 & 4.395 & 5 & 2.46 & 2.85 & 2.77 & 2.56 & 4.528 & 3 \\
\hline $\mathrm{Pd}$ & $\mathrm{E}$ & 2.63 & 2.64 & 2.59 & 2.64 & 4.515 & 5 & 2.46 & 2.53 & 2.76 & 2.53 & 4.650 & 8 \\
\hline $\mathrm{Ag}$ & $\mathrm{S}$ & 2.91 & 2.85 & 2.75 & 2.41 & 3.933 & 8 & 2.55 & 2.87 & 2.85 & 2.55 & 4.153 & 8 \\
\hline $\mathrm{Ag}$ & $\mathrm{E}$ & 2.69 & 2.69 & 2.65 & 2.69 & 4.050 & 1 & 2.51 & 2.58 & 2.82 & 2.58 & 4.326 & 2 \\
\hline $\mathrm{Ta}$ & $\mathrm{S}$ & 2.69 & 3.13 & 2.68 & 2.79 & 5.272 & 4 & 2.48 & 2.90 & 2.80 & 2.62 & 5.452 & 5 \\
\hline $\mathrm{Ta}$ & $\mathrm{E}$ & 2.78 & 2.59 & 2.69 & 2.59 & 5.350 & 9 & 2.49 & 2.56 & 2.80 & 2.56 & 5.483 & 10 \\
\hline W & $\mathrm{S}$ & 2.79 & 2.87 & 2.67 & 2.47 & 5.542 & 9 & 2.48 & 2.83 & 2.78 & 2.52 & 5.630 & 4 \\
\hline W & $\mathrm{E}$ & 2.72 & 2.56 & 2.65 & 2.56 & 5.620 & 9 & 2.47 & 2.52 & 2.77 & 2.52 & 5.663 & 10 \\
\hline $\mathrm{Pt}$ & $\mathrm{S}$ & 2.57 & 3.17 & 2.62 & 2.87 & 4.778 & 7 & 2.46 & 2.90 & 2.78 & 2.61 & 4.814 & 5 \\
\hline $\mathrm{Pt}$ & $\mathrm{E}$ & 2.63 & 2.71 & 2.61 & 2.71 & 4.857 & 5 & 2.46 & 2.59 & 2.78 & 2.59 & 4.926 & 8 \\
\hline $\mathrm{Au}$ & $S$ & 2.56 & 3.38 & 2.67 & 3.10 & 4.083 & 3 & 2.55 & 2.91 & 2.86 & 2.60 & 4.233 & 3 \\
\hline $\mathrm{Au}$ & $\mathrm{E}$ & 2.69 & 2.74 & 2.66 & 2.74 & 4.174 & 1 & 2.51 & 2.60 & 2.83 & 2.60 & 4.410 & 2 \\
\hline
\end{tabular}




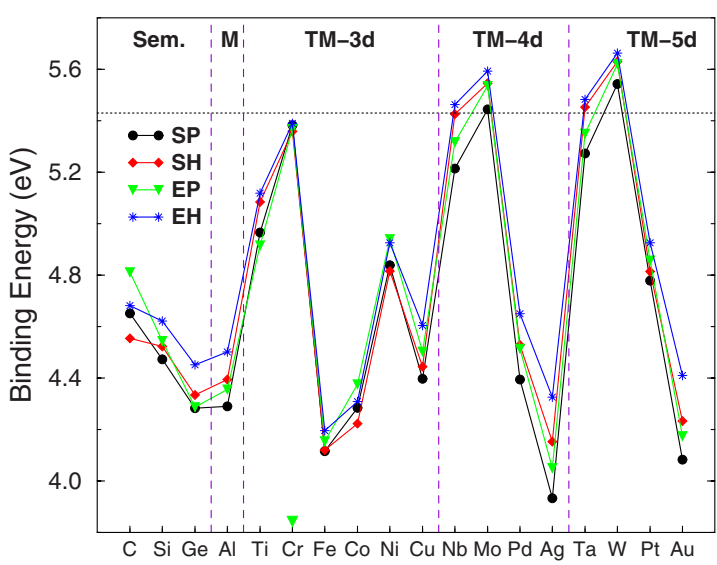

FIG. 3. (Color online) Comparison of the binding energies of the core-shell SiNWs doped with $M$ atoms $(M=\mathrm{C}, \mathrm{Si}, \mathrm{Ge}, \mathrm{Al}, \mathrm{Ti}, \mathrm{Cr}, \mathrm{Fe}, \mathrm{Co}, \mathrm{Ni}, \mathrm{Cu}, \mathrm{Nb}, \mathrm{Mo}, \mathrm{Pd}, \mathrm{Ag}, \mathrm{Ta}$, $\mathrm{W}, \mathrm{Pt}, \mathrm{Au}) . E_{b}$ of SP NWs is shown by black circles, of SH by red diamonds, of EP by green triangles and of EH by blue stars. Lines connecting these symbols are just for guide for the eyes only. Isolated green triangle for $\mathrm{Cr}$ is showing its binding energy in magnetic state. Horizontal dotted line shows the cohesive energy of bulk Si in diamond structure. Vertical dashed lines are just for separation of the regions for different types of dopant elements.

energy which is higher in energy by 0.049 and $0.014 \mathrm{eV}$, respectively, than that of the eclipsed one. For the cases of Ti and $\mathrm{Cr}$, the staggered pentagonal structure is more favorable because the bond lengths of $\mathrm{Ti}-\mathrm{Ti}\left(\ell_{\mathrm{Ti}-\mathrm{Ti}}=2.06 \AA\right)$ and $\mathrm{Cr}-\mathrm{Cr}$ $\left(\ell_{\mathrm{Cr}-\mathrm{Cr}}=2.15 \AA\right)$ are smaller than that of other elements. Similarly, all eclipsed hexagonal structures are also energetically more favorable than corresponding staggered ones (see Table I and Fig. 3). However, for all of the elements under consideration, the differences in binding energies of eclipsed and staggered wires are generally small and range between 5 to $200 \mathrm{meV}$ for both pentagonal and hexagonal structures.

Furthermore, the energetic behaviors of pentagonal and hexagonal eclipsed and staggered structures of core-shell SiNWs are illustrated in Fig. 3. According to the comparison of the binding energies of SiNWs doped with different $M$ atoms $(M=\mathrm{C}, \mathrm{Si}, \mathrm{Ge}, \mathrm{Al}, \mathrm{Ti}, \mathrm{Cr}, \mathrm{Fe}, \mathrm{Co}$, $\mathrm{Ni}, \mathrm{Cu}, \mathrm{Nb}, \mathrm{Mo}, \mathrm{Pd}, \mathrm{Ag}, \mathrm{Ta}, \mathrm{W}, \mathrm{Pt}, \mathrm{Au})$ as presented in Fig. 3, it is seen that the eclipsed hexagonal structure of SiNW enriched by one additional dopant atom lying along the wire axis as a linear chain has the highest averaged binding energy compared to the other structures considered in this study, even though the $E_{b}$ 's of all four wire structure are very close to each other. However, for the cases of $\mathrm{C}, \mathrm{Ni}$, and $\mathrm{Co}$, lower energy geometry is the eclipsed pentagonal structure. But for the $\mathrm{C}$ dopant $\mathrm{EH}$ wires, there are two different metastable structures, the one with nonuniform bond lengths has actually the highest binding energy (see also Table I). It is clear that the hexagonal geometry is the lowest in energy and $M$ atoms fastened to the linear chain passing through the center of hexagonal geometry provides optimal bounding with the Si-shell atoms. Moreover, it is useful to compare the averaged binding energies with the calculated bulk binding energy of diamond structure of $\mathrm{Si}$, which is $5.43 \mathrm{eV}$. It is seen that the averaged binding energies of core-shell SiNWs
TABLE II. Magnetic moment (in terms of $\mu_{B}$ ) of core-shell SiNWs doped with $\mathrm{Fe}, \mathrm{Co}$, or $\mathrm{Cr}$ atoms at the core.

\begin{tabular}{lccc}
\hline \hline Structure & $\mathrm{Fe}$ & $\mathrm{Co}$ & $\mathrm{Cr}$ \\
\hline $\mathrm{EP}$ & 1.76 & 0.60 & 0.22 \\
$\mathrm{SP}$ & 3.08 & 2.12 & \\
$\mathrm{EH}$ & 2.68 & 1.35 & \\
$\mathrm{SH}$ (nonuniform) & 4.09 & 1.58 & \\
$\mathrm{SH}$ (uniform) & & 1.48 & \\
\hline \hline
\end{tabular}

presented in Table I are usually lower than the bulk cohesive energy. This can be explained by higher coordination number in bulk crystals. According to the well-known general trend, the binding energy decreases with decreasing coordination number in different structures. ${ }^{44,45}$ For transition metal dopants, along the rows of periodic table, $E_{b}$ of SiNWs first increases making a peak at group VI elements and then decreases. Actually, for the cases of $\mathrm{Nb}, \mathrm{Mo}, \mathrm{Ta}$, and $\mathrm{W}$, it exceeds the cohesive energy of diamond $\mathrm{Si}$. For Fe, Co, and $\mathrm{Cr}$, spin-polarized calculations yield a magnetic ground state, but the $E_{b}$ of these nanowires are lower compared to other nonmagnetic wires.

According to spin-polarized calculations, it is seen that SiNWs doped with magnetic transition metals $\mathrm{Fe}, \mathrm{Co}$, and $\mathrm{Cr}$ exhibit magnetic moments (in $\mu_{B}$ ) as presented in Table II. The calculated magnetic moment per atom of Fe-doped eclipsed hexagonal SiNW is nearly $2.67 \mu_{B}$. This value is slightly larger than the magnetic moment value of the bulk Fe crystal $\left(2.22 \mu_{B}\right)$. This means that Fe-doped eclipsed hexagonal structure of SiNWs is more magnetic than in a pure metallic form. Based on the previous calculations for doped infinite nanotubes $\left(2.4 \mu_{B}\right),{ }^{50}$ it is seen that Fe-doped eclipsed hexagonal SiNW has a higher magnetic moment. Other forms of Fe-doped SiNWs under considerations exhibit lower magnetic moments than that of the eclipsed hexagonal structure per Fe atom (see Table II) but larger than the previous result for finite nanotube $\left(1.7 \mu_{B}\right) \cdot{ }^{50}$ For Co doping case, the magnetic moment in the eclipsed hexagonal structure which is the energetically most stable geometry is nearly $1.35 \mu_{B}$ and smaller than that of the bulk metal $\left(1.72 \mu_{B}\right)$. In the case of $\mathrm{Cr}$, the magnetic moment per $\mathrm{Cr}$ atom is only $0.22 \mu_{B}$ for the eclipsed pentagonal structure. The above results for the magnetic moments imply that the doping mechanism with magnetic transition metal atoms causes direct interactions between magnetic ions and this is essential for maintaining strong magnetism, mainly one dimensional.

\section{B. Electronic band structures}

It has been well known that the number of conductance channels is determined by the number of electronic bands crossing the Fermi level $\left(E_{F}\right)$ and sensitively depends on the atomic structure of a nanowire, which can be regarded as an atomic sized constriction between two electron reservoirs. If the size of a nanowire is comparable to the Fermi wavelength of the conducting electrons, the electrical conduction $(G)$ can be quantized according to the Landauer equation 

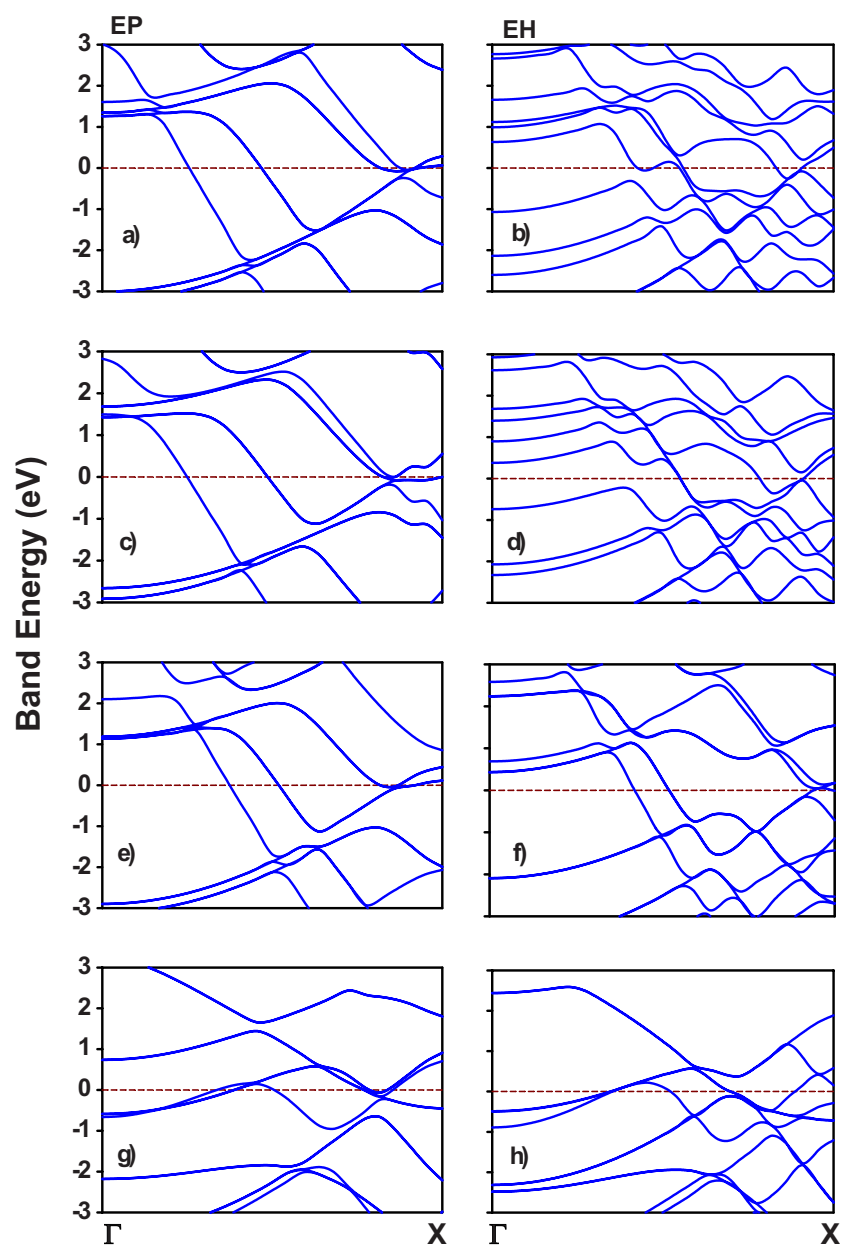

FIG. 4. (Color online) Energy band structures of EP and EH configurations of core-shell SiNWs doped with selected $M$ atoms. (a) and (b) for Si core; (c) and (d) for Ge core; (e) and (f) for $\mathrm{Al}$ core; (g) and (h) for Ti core. The zeros of the energy are set at the Fermi level represented by dashed lines.

$G=G_{0} \sum_{n \sigma} T_{n \sigma}$, where $G_{0}=e^{2} / h$ is the conductance quantum ( $e$ is the electronic charge, $h$ is Planck's constant), $T_{n \sigma}$ is a transmission coefficient for the $n^{\text {th }}$ channel and $\sigma$ is electron spin, which can take one of two values either up( $\uparrow)$ or down $(\downarrow)$. For the quantum ballistic transport, $T_{n \sigma}$ can either be 1 or 0 corresponding to an open or closed channel. In the case of nonmagnetic materials, the electron transport is ballistic along the transverse direction forming the well-defined quantum channels and each channel contributes equally to the conductance by assuming a perfect contact. The conductance becomes, thus, quantized and is given by $G_{0}=2 e^{2} / h$, where 2 comes from the spin directions. Along these lines, since the conductance depends on the contacts, we examined the transport properties of core-shell SiNWs from the number of electronic bands crossing the Fermi level from electronic band structure calculations and they are summarized in Table I.

Our analysis shows that the electronic band structures of all core-shell SiNWs doped with $M$ atoms have band crossing at Fermi level for both magnetic and nonmagnetic atoms at the core. According to these characters of bands, all of geometric structures of SiNWs (i.e., EP, SP, EH, SH) doped
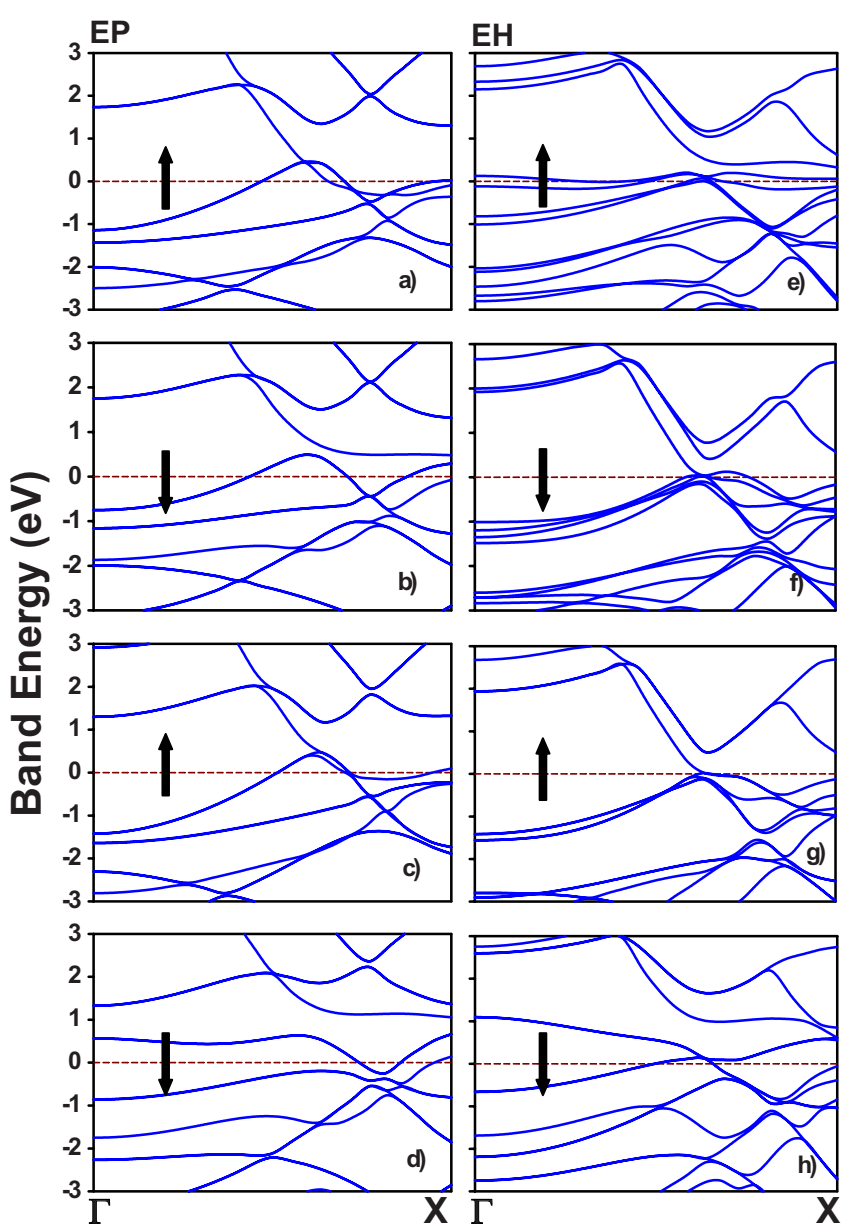

FIG. 5. (Color online) The spin-up and spin-down band structures of Co- and Fe-doped EP and EH structures of SiNWs. (a), (b), (e) and (f) for $\mathrm{Co}$; (c), (d), (g), and (h) for Fe. The Fermi levels are represented by dashed lines and lie at the zero of energy.

with $M$ atoms indicate metallic behavior with calculated conductance channel numbers compiled in Table I. Although the same kinds of structures have the same number of atoms in their unit cells, the number of electronic bands crossing the Fermi level is not the same for the different $M$ atoms at the core. A comparative analysis of the electronic band structures of selected $M$ atoms, $\mathrm{Si}, \mathrm{Ge}, \mathrm{Al}$, Ti, core atoms is illustrated in Fig. 4, displaying the Fermi level by dashed lines which are set to zero. In the case of Si doped SiNWs as shown in Figs. 4(a) and 4(b), the number of bands crossing the Fermi level is 10 and 6 for EP and EH structures, respectively. For the staggered ones as shown in Table I, but doesn't appear in Fig. 4, this number is 6 and 4, respectively. Comparison of the previous results about the pentagonal nanowires of $\mathrm{Si}$ investigated by Sen et ll $^{45}$ using similar first-principles calculation methods shows that our results for specific structures, EP and SP, which are common for SiNWs in both studies, are in good agreement. For Ge as shown in Fig. 4(c) and $4(\mathrm{~d})$, the number of bands for the eclipsed (staggered) pentagonal and hexagonal structures is 10 (6) and 4 (9), respectively. One of the interesting result is that the staggered hexagonal structure has almost the same number of bands with the eclipsed pentagonal one. This means that both struc- 
tures give rise to high density of states at the close vicinity of the Fermi level. In the cases of $\mathrm{Al}$ and $\mathrm{Ti}$ given in Figs. 4(e)-4(h), respectively, the EP structures have nine and ten bands, respectively, crossing the Fermi level whereas the hexagonal ones contain five and seven bands. Note that some bands below the Fermi energy are dropped for the eclipsed structures being more stable because these structures have a weak bond formation between $\mathrm{Si}$-shell atoms on different pentagonal and hexagonal planes. ${ }^{45}$

The number of bands of core-shell SiNWs doped with Fe, $\mathrm{Co}$, and $\mathrm{Cr}$ atoms (with the spin-polarized calculation) depends on the calculated occupation number of electrons for each spin direction. Hence, as shown in Table I, the total number of bands crossing the Fermi level for Fe-doped structures of SiNWs is nine $[6(\uparrow)+3(\downarrow)]$ and six $[4(\uparrow)+2(\downarrow)]$ for EP and SH structures, respectively. This number is seven $[3(\uparrow)+4(\downarrow)]$ for SP and EH structures. For Co, the sum of bands at the Fermi level is thirteen $[8(\uparrow)+5(\downarrow)]$ for the EH structure which has the largest spin polarization. In the case of Cr-doped SiNWs, the calculated magnetic moment is weakly $0.22 \mu_{B}$ for the EP structure but other structures of SiNWs doped with $\mathrm{Cr}$ atom have not any magnetic moment value. This is also valid for $\mathrm{Ni}$ and $\mathrm{Ti}$ cases and is consistent with the argument that low magnetic moments may be quenched by even a weaker hybridization. ${ }^{55,56}$ In Fig. 5, the spin-up and spin-down band structures of Co- and Fe-doped SiNWs are shown. Figures 5(a) and 5(b) and Fig. 5(e) and $5(\mathrm{f})$ indicate the spin magnetic behavior of the Co-doped EP and EH structures of SiNWs, respectively. Other parts of Fig. 5 belong to Fe-doped cases. Note that most of the bands crossing at the Fermi level in Fig. 5 are nearly degenerate. In all cases of $\mathrm{Co}$ and $\mathrm{Fe}$ and the $\mathrm{EP}$ case of $\mathrm{Cr}$, there is different number of band crossing at the Fermi level for both the spin-up and spin-down components, indicating ferromagnetic behavior. This may be an important aspect for spintronics as well as other nanoscale magnetic applications.

\section{CONCLUSIONS}

In summary, we have presented extensive first-principles calculations on the structural stabilities and electronic band structures of core-shell SiNWs doped with several $M$ atoms at the core such as semiconductors, simple metal, and transition metals. Especially, we have studied eclipsed and staggered structures made from pentagons and hexagons. We have seen that all geometric structures of core-shell SiNWs doped with $M$ atoms exhibit metallic behavior. According to the stability and energetic analysis of SiNWs, we have found that EP and EH structures are the energetically more stable than staggered ones. However, there are exceptions. For instance, staggered pentagonal structures for Cr-doped SiNWs are found to be energetically degenerate with the eclipsed one. An important aspect of the doping of $3 d$ transition metals is the magnetic behavior. From this point view, we have predicted that $\mathrm{Co}-, \mathrm{Fe}$ - and $\mathrm{Cr}$ (in magnetic form)-doped structures of SiNWs are spin polarized. Fe-doped SiNW has large magnetic moment per Fe atom for the $\mathrm{EH}$ structure. Hence, further experimental studies on pentagonal and hexagonal core-shell silicon nanowires which might have an important role in spintronics devices and nanoelectronics will be beneficial.

\section{ACKNOWLEDGMENTS}

We acknowledge support from the Scientific and Technological Research Council of Turkey (TÜBİTAK) under Program Code. 2218 and Project Grant No. 107T011, as well as partial support from Erciyes University under Project Grant No. FBA-07-01 and Bilkent University. Some parts of the computations have been performed using the ULAKBIM High Performance Computer Center. O.G. acknowledges the support of Turkish Academy of Sciences, TÜBA. *gulseren@fen.bilkent.edu.tr

${ }^{1}$ Y. Cui and C. M. Lieber, Science 291, 851 (2001).

${ }^{2}$ N. Mathur, Nature (London) 419, 573 (2002).

${ }^{3}$ R. S. Friedman, M. C. McAlpine, D. S. Ricketts, D. Ham, and C. M. Lieber, Nature (London) 434, 1085 (2005).

${ }^{4}$ M. Xiao, I. Martin, E. Yablonovitch, and H. W. Jiang, Nature (London) 430, 435 (2004).

${ }^{5}$ D. D. D. Ma, C. S. Lee, F. C. K. Au, S. Y. Tong, and S. T. Lee, Science 299, 1874 (2003).

${ }^{6}$ J. L. Liu, Y. Lu, Y. Shi, S. L. Gu, R. L. Jiang, F. Wang, and Y. D. Zheng, Appl. Phys. A: Mater. Sci. Process. 66, 539 (1998).

${ }^{7}$ J. Hu, M. Ouyang, P. Yang, and C. M. Lieber, Nature (London) 399, 48 (1999).

${ }^{8}$ M. S. Gudiksen, L. J. Lauhon, J. Wang, D. C. Smith, and C. M. Lieber, Nature (London) 415, 617 (2002).

${ }^{9}$ S. Christiansen and U. Gösele, Nature Mater. 3, 357 (2004).

${ }^{10}$ A. Colli, S. Hofmann, A. Fasoli, A. C. Ferrari, C. Ducati, R. E. Dunin-Borkowski, and J. Robertson, Appl. Phys. A: Mater. Sci. Process. 85, 247 (2006).
${ }^{11}$ G. Zheng, W. Lu, S. Jin, and C. M. Lieber, Adv. Mater. 16, 1890 (2004).

${ }^{12}$ J. Goldberger, A. I. Hochbaum, R. Fan, and P. Yang, Nano Lett. 6, 973 (2006).

${ }^{13}$ X. Duan, C. Niu, V. Sahi, J. Chen, J. W. Parce, S. Empedocles, and J. L. Goldman, Nature (London) 425, 274 (2003).

${ }^{14}$ Y. Wu, J. Xiang, C. Yang, W. Lu, and C. M. Lieber, Nature (London) 430, 61 (2004).

${ }^{15}$ L. Canham, Nature (London) 408, 411 (2000).

${ }^{16}$ S. M. Koo, M. D. Edelstein, Q. Li, C. A. Richter, and E. M. Vogel, Nanotechnology 16, 1482 (2005).

${ }^{17}$ U. Landman, R. N. Barnett, A. G. Scherbakov, and P. Avouris, Phys. Rev. Lett. 85, 1958 (2000).

${ }^{18}$ J. Bai, X. C. Zeng, H. Tanaka, and J. Y. Zeng, Proc. Natl. Acad. Sci. U.S.A. 101, 2664 (2004).

${ }^{19}$ G. Seifert, Th. Köhler, H. M. Urbassek, E. Hernández, and Th. Frauenheim, Phys. Rev. B 63, 193409 (2001).

${ }^{20}$ M. Zhang, Y. H. Kan, Q. J. Zang, Z. M. Su, and R. S. Wang, Chem. Phys. Lett. 379, 81 (2003). 
${ }^{21}$ E. Durgun, S. Tongay, and S. Ciraci, Phys. Rev. B 72, 075420 (2005).

${ }^{22}$ X. Yang and J. Ni, Phys. Rev. B 72, 195426 (2005).

${ }^{23}$ A. S. Barnard and S. P. Russo, J. Phys. Chem. B 107, 7577 (2003).

${ }^{24}$ N. Wang, Y. H. Tang, Y. F. Zhang, C. S. Lee, and S. T. Lee, Phys. Rev. B 58, R16024 (1998).

${ }^{25}$ S. B. Fagan, R. J. Baierle, R. Mota, A. J. R. da Silva, and A. Fazzio, Phys. Rev. B 61, 9994 (2000).

${ }^{26}$ E. Durgun, D. Çakır, N. Akman, and S. Ciraci, Phys. Rev. Lett. 99, 256806 (2007).

${ }^{27}$ E. Durgun, N. Akman, C. Ataca, and S. Ciraci, Phys. Rev. B 76, 245323 (2007).

${ }^{28}$ M.-F. Ng, L. Zhou, S.-W. Yang, L. Y. Sim, V. B. C. Tan, and P. Wu, Phys. Rev. B 76, 155435 (2007).

${ }^{29}$ P. B. Sorokin, P. V. Avramov, A. G. Kvashnin, D. G. Kvashnin, S. G. Ovchinnikov, and A. S. Fedorov, Phys. Rev. B 77, 235417 (2008).

${ }^{30} \mathrm{X}$. Blase and M.-V. Fernandez-Serra, Phys. Rev. Lett. 100, 046802 (2008).

${ }^{31}$ J. X. Cao, X. G. Gong, J. X. Zhong, and R. Q. Wu, Phys. Rev. Lett. 97, 136105 (2006).

${ }^{32}$ P. W. Leu, B. Shan, and K. Cho, Phys. Rev. B 73, 195320 (2006).

${ }^{33}$ I. Ponomareva, M. Menon, D. Srivastava, and A. N. Andriotis, Phys. Rev. Lett. 95, 265502 (2005); I. Ponomareva, M. Menon, E. Richter, and A. N. Andriotis, Phys. Rev. B 74, 125311 (2006).

${ }^{34}$ J. Li and A. J. Freeman, Phys. Rev. B 74, 075333 (2006).

${ }^{35}$ B.-X. Li, P.-L. Cao, R. Q. Zhang, and S. T. Lee, Phys. Rev. B 65, 125305 (2002).

${ }^{36}$ Y. Zhao and B. I. Yakobson, Phys. Rev. Lett. 91, 035501 (2003).

${ }^{37}$ Y. Zheng, C. Rivas, R. Lake, K. Alam, T. B. Boykin, and G. Klimeck, IEEE Trans. Electron Devices 52, 1097 (2005).

${ }^{38}$ A. K. Singh, V. Kumar, R. Note, and Y. Kawazoe, Nano Lett. 6, 920 (2006)

${ }^{39}$ B. Marsen and K. Sattler, Phys. Rev. B 60, 11593 (1999).

${ }^{40}$ R. Kagimura, R. W. Nunes, and H. Chacham, Phys. Rev. Lett. 95, 115502 (2005).

${ }^{41}$ M. Durandurdu, Phys. Status Solidi B 243, R7 (2006).

${ }^{42}$ G. B. Adams, M. O'Keeffe, A. A. Demkov, O. F. Sankey, and
Y.-M. Huang, Phys. Rev. B 49, 8048 (1994).

${ }^{43}$ S. Sirichantaropass, V. M. García-Suárez, and C. J. Lambert, Phys. Rev. B 75, 075328 (2007).

${ }^{44}$ O. Gülseren, F. Ercolessi, and E. Tosatti, Phys. Rev. Lett. 80, 3775 (1998).

${ }^{45}$ P. Sen, O. Gülseren, T. Yildirim, I. P. Batra, and S. Ciraci, Phys. Rev. B 65, 235433 (2002).

${ }^{46}$ H. Hiura, T. Miyazaki, and T. Kanayama, Phys. Rev. Lett. 86, 1733 (2001).

${ }^{47}$ V. Kumar and Y. Kawazoe, Phys. Rev. Lett. 87, 045503 (2001).

${ }^{48}$ S. N. Khanna, B. K. Rao, and P. Jena, Phys. Rev. Lett. 89, 016803 (2002).

${ }^{49}$ J. Lu and S. Nagase, Phys. Rev. Lett. 90, 115506 (2003).

${ }^{50}$ V. Kumar and Y. Kawazoe, Phys. Rev. Lett. 90, 055502 (2003).

${ }^{51}$ L.-J. Guo, G.-F. Zhao, Y.-Z. Gu, X. Liu, and Z. Zeng, Phys. Rev. B 77, 195417 (2008).

${ }^{52}$ M. Menon, A. N. Andriotis, and G. E. Froudakis, Nano Lett. 2, 301 (2002).

${ }^{53}$ A. N. Andriotis, G. Mpourmpakis, G. E. Froudakis, and M. Menon, New J. Phys. 4, 78 (2002).

${ }^{54}$ T. Dumitrică, M. Hua, and B. I. Yakobson, Phys. Rev. B 70, 241303(R) (2004).

${ }^{55}$ A. K. Singh, T. M. Briere, V. Kumar, and Y. Kawazoe, Phys. Rev. Lett. 91, 146802 (2003).

${ }^{56}$ Y.-R. Jang, C. Jo, and J. I. Lee, IEEE Trans. Magn. 41, 3118 (2005).

${ }^{57}$ P. Hohenberg and W. Kohn, Phys. Rev. 136, B864 (1964); W. Kohn and L. J. Sham, ibid. 140, A1133 (1965).

${ }^{58}$ M. C. Payne, M. P. Teter, D. C. Allen, T. A. Arias, and J. D. Joannopoulos, Rev. Mod. Phys. 64, 1045 (1992).

${ }^{59}$ Computations have been carried out by using the VASP software: G. Kresse and J. Hafner, Phys. Rev. B 47, 558 (1993); G. Kresse and J. Furthmüller, ibid. 54, 11169 (1996).

${ }^{60}$ D. Vanderbilt, Phys. Rev. B 41, 7892 (1990).

${ }^{61}$ J. P. Perdew and A. Zunger, Phys. Rev. B 23, 5048 (1981).

${ }^{62}$ J. P. Perdew and Y. Wang, Phys. Rev. B 45, 13244 (1992); J. P. Perdew, J. A. Chevary, S. H. Vosko, K. A. Jackson, M. R. Pederson, D. J. Singh, and C. Fiolhais, ibid. 46, 6671 (1992).

${ }^{63}$ M. Methfessel and A. T. Paxton, Phys. Rev. B 40, 3616 (1989).

${ }^{64}$ H. J. Monkhorst and J. D. Pack, Phys. Rev. B 13, 5188 (1976). 